\title{
METRIC PROPERTIES OF THE LAMPLIGHTER GROUP AS AN AUTOMATA GROUP
}

\author{
SEAN CLEARY AND JENNIFER TABACK
}

\begin{abstract}
We develop the geometry of the Cayley graph of the lamplighter group with respect to the generating set rising from its interpretation as an automata group 4. We find metric behavior with respect to this generating set analogous to the metric behavior in the standard group theoretic generating set. This includes expressions for normal forms and geodesic paths, and families of 'dead-end' words and 'seesaw' words.
\end{abstract}

\section{INTRODUCTION}

There are several generating sets of interest for the lamplighter groups $L_{n}$, which are wreath products of finite cyclic groups with the integers. The standard presentation of $L_{n}$ arises from the wreath product structure, namely

$$
L_{n}=\left\langle a, t \mid\left[t^{i} a t^{-i}, t^{j} a t^{-j}\right], a^{n}\right\rangle .
$$

For convenience, we will refer to the elements $\{a, t\}$ as the wreath product generators of $L_{n}$. The lamplighter group $L_{2}$ can also be regarded as an automata group [4, that is, isomorphic to a group generated by a finite collection of finite state automata. The natural generating set arising from this interpretation is $\{a, t a\}$, where $a$ and $t$ are the wreath product generators of $L_{2}$. We will refer to the generating set $\{a, t a\}$ as the automata generating set of $L_{2}$. Grigorchuck and Zuk compute the spectral radius of $L_{2}$ with respect to this generating set; they find remarkably that it is a discrete measure.

We explored the geometry of the Cayley graph of $L_{n}$ with respect to the wreath product generating set $\{a, t\}$ in [1. Here we describe similar aspects of the geometry of these groups with respect to the automata generating set.

\section{Normal fOrms AND GeOdesics IN $L_{2}$}

As described in [1], we consider a word in $L_{2}=\mathbb{Z}_{2} \prec \mathbb{Z}$ geometrically as a bi-infinite string of light bulbs together with a cursor. Each bulb has two states, on and off, and the cursor which indicates the current bulb under consideration. A word in the lamplighter group $L_{2}$ begins with a bi-infinite string of bulbs all in the off state, and is a sequence of

\footnotetext{
The first author acknowledges support from PSC-CUNY grant \#64459-0033.

The second author acknowledges support from NSF grant \#0305441, and would like to thank Bowdoin College for their hospitality during the writing of this paper.
} 


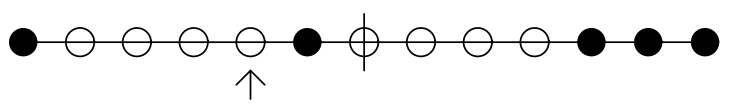

Figure 1. A typical element of the group $L_{2}, w=a_{4} a_{5} a_{6} a_{-1} a_{-6} t^{-2}$. Solid circles represent bulbs which are on, open circles represent bulbs which are off, the vertical bar denotes the origin in $\mathbb{Z}$, and the arrow denotes the final position of the cursor.

movements of the cursor and commands to illuminate a finite collection of bulbs. The identity word is represented by the configuration of bulbs which are all in the off state, and the cursor at the origin. Figure 1 gives an example of a word in $L_{2}$ represented as a bi-infinite string of light bulbs along with a cursor.

The wreath product generators $a$ and $t$ of $L_{2}$ encapsulate the two instructions necessary to create a word $w \in L_{2}$. The generator $t$ moves the cursor one step to the right, $t^{-1}$ moves the cursor one step to the left, and $a=a^{-1}$ changes the state of the bulb at the current location of the cursor. The automata generators of $L_{2}$ combine these two operations. The generator $t a$ moves the cursor one step to the right and changes the state of the bulb at the new location of the cursor, and its inverse $(t a)^{-1}=a t^{-1}$ changes the state of the bulb at the current location and then moves the cursor one step to the left.

The final location of the cursor in a word $w \in L_{2}$ is easily computed as the exponent sum of $t$ if the word is described in terms of the wreath product generating set or as the exponent sum of $t a$ if the word is described in the automata generating set.

2.1. Normal forms. We would like to be able to calculate the word length of $w \in L_{2}$ with respect to the automata generating set, analogous to the computation of word length with respect to the wreath product generating set presented in 1]. This was accomplished in [1] through the use of normal forms for elements of $L_{2}$. Below, we use the same normal forms to aid us in computing the word length of $w$ with respect to the automata generating set.

Normal forms for elements of $L_{2}$ with respect to the wreath product generators $a$ and $t$ are given in terms of the conjugates $a_{k}=t^{k} a t^{-k}$, which move the cursor to the $k$-th bulb, turn it on, and return the cursor to the origin. We note that we can also consider $a_{k}$ as the conjugate of $a$ by $t a$, since $a_{k}=(t a)^{k} a(t a)^{-k}$ in $L_{2}$.

In [1, we present two normal forms for an element $w \in L_{2}$, the right first normal form given by

$$
r f(w)=a_{i_{1}} a_{i_{2}} \ldots a_{i_{m}} a_{-j_{1}} a_{-j_{2}} \ldots a_{-j_{l}} t^{r}
$$

and the left first normal form given by

$$
l f(w)=a_{-j_{1}} a_{-j_{2}} \ldots a_{-j_{l}} a_{i_{1}} a_{i_{2}} \ldots a_{i_{m}} t^{r}
$$

with $i_{m}>\ldots i_{2}>i_{1} \geq 0$ and $j_{l}>\ldots j_{2}>j_{1}>0$. In the right first normal form, the cursor first moves towards the right, lighting the appropriate bulbs, then moves to the 
left, lighting bulbs to the left of the origin, and lastly moves to its final position. The left first normal form does the reverse.

One or possibly both of these normal forms will lead to a minimal length representative for $w \in L_{2}$ with respect to the wreath product generating set, depending upon the final location of the cursor relative to the origin. That position is easy to detect from the sign of the exponent sum of $t$, given as $r$ above.

We now describe when one of these normal forms will yield a minimal length representative for an element with respect to the automata generating set $\{a, t a\}$. We do this for words $w$ with non-negative exponent sum on the generator $t$ when written in either normal form above; for these words the left first normal form will produce a minimal length representative with respect to the wreath product generating set. The case of words with negative exponent sum on the generator $t$ is completely analogous and uses right first normal form. We now consider a word $w$ with non-negative exponent sum on the generator $t$ in the left first normal form, and divide our words into two types depending on the final position of the cursor, as follows.

Type I: $w$ is a word in which the final position of the cursor is to the right of the origin and at or to the right of the rightmost illuminated bulb.

Type II: $w$ is a word in which the final position of the cursor is at or to the right of the origin and to the left of the rightmost illuminated bulb. In type II words we subdivide the illuminated positive bulbs into two groups:

- bulbs in positions $i_{1}, \ldots, i_{s}$ up to and possibly including the bulb at the final resting position of the cursor, and

- bulbs in positions $i_{s+1}, \ldots, i_{m}$ which are to the right of the final position of the cursor.

In [1, we used the normal form to compute the word length of $w \in L_{2}$ with respect to the wreath product generators.

Proposition 2.1 (Proposition 3.6 [1]). Let $w \in L_{2}$ be in either normal form, and define

$$
D(w)=m+l+\min \left\{2 j_{l}+i_{m}+\left|r-i_{m}\right|, 2 i_{m}+j_{l}+\left|r+j_{l}\right|\right\} .
$$

The word length of $w$ with respect to the generating set $\{a, t\}$ is exactly $D(w)$.

We now define a related quantity $D^{\prime}(w)$, also dependent on the normal form of $w$, which will give the word length of $w$ with respect to the automata generating set.

Definition 2.2. Let $w \in L_{2}$ be a word with non-negative exponent sum on the generator $t$, considered in left first normal form.

If $w$ is Type I, define $D^{\prime}(w)=2 j_{l}+l+2 r-m$.

If $w$ is Type II, define $D^{\prime}(w)=2 j_{l}+l+2 i_{m}+m-2 s$. 
We now prove that the quantity $D^{\prime}(w)$, which is computed from the normal form of $w$ in the wreath product generating set, yields the word length of $w$ with respect to the automata generating set. We first note that a minimal length representative for $w$ in the wreath product generating set will have the same exponent sum on $t$ as the exponent sum of $t a$ in a minimal length representative for $w$ with respect to the automata generating set. This follows because the generator $a$ in either generating set cannot change the position of the cursor. Since we are considering word length with respect to the automata generating set below, we phrase Proposition 2.3 in terms of the exponent sum of $t a$.

Proposition 2.3. Let $L_{2}$ be generated by a and ta, as given above. The word length of $w \in L_{2}$ with non-negative ta exponent sum with respect to the automata generating set is given by $D^{\prime}(w)$.

It follows immediately that we can use $D^{\prime}(w)$ to calculate the word length of any $w \in L_{2}$ with respect to the automata generating set, since $|w|=\left|w^{-1}\right|$, and one of these two elements will have non-negative $t a$ exponent sum.

Proposition 2.3 is proved via the following two lemmas.

Lemma 2.4. The length of a word $w \in L_{2}$ with non-negative ta exponent sum with respect to the generating set $\{a, t a\}$ is at most $D^{\prime}(w)$.

Proof. We proceed similarly to [1, where the word length is computed with respect to the wreath product generating set. First we write $w$ in terms of $a$ and $t a$ in the order suggested by the left-first normal forms, using the fact that $a_{k}=t^{k} a t^{-k}$ can also be written $a_{k}=(t a)^{k} a(t a)^{-k}$. Counting instances of $a$ and $t a$ after cancellation, one exactly obtains $D^{\prime}(w)$, and thus the word length of $w$ with respect to the automata generating set is at least $D^{\prime}(w)$.

Lemma 2.5. The length of a word $w \in L_{2}$ with non-negative ta exponent sum with respect to the generating set $\{a, t a\}$ is at least $D^{\prime}(w)$.

Proof. As when computing the word length of $w$ with respect to the wreath product generating set, we consider the minimal number of generators required for moving the cursor and illuminating bulbs to create the word $w$.

This count reflects the fact that the generator $t a$ in automata generating set combines the two basic 'operations' of moving the cursor and illuminating a bulb. Because of this, bulbs which are visited an odd number of times by the cursor as a word is constructed are left illuminated. If the word requires that bulb to be turned off, an extra application of the generator $a$ is required to turn off the bulb. If a bulb in a certain position is visited an even number of times by the cursor, then the default state of the bulb is off, and if the word requires that bulb illuminated, an extra application of the generator $a$ is required again.

Thus when counting the minimal number of generators necessary to construct a word $w \in L_{2}$ which is of type II, we need to count the number of extra $a$ generators necessary 
to turn off the bulbs in positive positions to the left of the final cursor position, and the number of extra $a$ generators necessary to turn on the bulbs to the right of the final cursor position.

Case I: First, we consider a word $w$ with non-negative ta exponent sum which is Type I, so the final cursor position lies to the right of all illuminated bulbs, in the left first normal form. We use the fact that for any prefix of any representative of the word $w$, the exponent sum of the generator $t a$ yields the current position of the cursor.

If $w=a_{-j_{1}} a_{-j_{2}} \ldots a_{-j_{l}} a_{i_{1}} a_{i_{2}} \ldots a_{i_{m}} t^{r}$, we consider the minimal number of instances of the generator $t a$ required to construct $w$. To turn on the leftmost illuminated bulb, which we see from the normal form is in position $-j_{l}$, a minimal representative for $w$ must have a prefix with exponent sum $-j_{l}$. Similarly, the total exponent sum of $t a$ in any representative for $w$ must be $r$, since that is the final position of the cursor. Thus, any word representing $w$ must have at least $j_{l}+j_{l}+r$ occurrences of $t a$.

Let $\gamma$ be any string of generators in the automata generating set which represents the word $w$. We consider the number of times bulbs in different positions will be visited by the cursor as proscribed by the string of generators. Since the cursor starts at the origin and ends at a nonnegative final position in $w$, we conclude the following.

- Bulbs at and to left of the origin will be visited an even number of times

- Bulbs to the right of the origin and at or to the left of the final cursor position will be visited an odd number of times.

- Bulbs to the right of the final cursor position will be visited an even number of times. For minimal length representatives, these bulbs will be visited zero times.

When the left first normal form for $w$ is written using $a_{k}=(t a)^{k} a(t a)^{-k}$, the bulbs in positions $-j_{l}$ through 0 will be visited twice and bulbs 1 through $i_{m}$ will be visited once, but there are other strings of generators representing $w$ which may visit these bulbs more often than that and may visit other bulbs as well. However, the parity of the number of visits is completely determined.

Thus, for any representative for $w$, there will need to be at least $l$ occurrences of the generator $a$ to turn on all required bulbs at the origin and to its left, and at least $r-m$ occurrences of $a$ to turn off bulbs to the right of the origin which otherwise would have been left on. Counting the total number of required $t a$ 's and $a$ 's, we obtain $D^{\prime}(w)$, which is thus a lower bound for the word length of $w$.

Case II: Now we consider a word $w$ with non-negative $t a$ exponent sum where the final cursor position lies to the left of the rightmost illuminated bulb, with normal form as described above. Again we notice that any string $\gamma$ of generators representing $w$ must have a prefix with $t a$ exponent sum $-j_{l}$, since the leftmost illuminated bulb is in position $-j_{l}$. To turn on the rightmost bulb in position $i_{m}$, the string $\gamma$ must have a prefix with ta exponent sum $i_{m}$. Finally, for the cursor to be left at position $r$, the final ta exponent sum must be $r$. Thus $\gamma$ must have at least $j_{l}+j_{l}+i_{m}+\left(i_{m}-r\right)$ occurrences of the generator $t a$ to accomplish this movement of the cursor. 
We again consider the number of times bulbs in different positions will be visited by the cursor as proscribed by the string $\gamma$ of generators.

- Bulbs at and to left of the origin will be visited an even number of times.

- Bulbs to the right of the origin and at or to the left of the final cursor position will be visited an odd number of times.

- Bulbs to the right of the final cursor position will be visited an even number of times.

When the left first normal form for $w$ is written using $a_{k}=(t a)^{k} a(t a)^{-k}$, the bulbs $-j_{l}$ through 0 will be visited twice and bulbs 1 through $i_{s}$ will be visited once, and bulbs $i_{s+1}$ to $i_{m}$ will be visited twice. In other strings of generators representing $w$, other bulbs may be visited by the cursor, but the parity of the number of visits is again determined.

When computing the length of a minimal representative of $w$ in the automata generating set, the number of occurrences of the generator $a$ will be the number of bulbs left on to the left of the origin $l$, the number of bulbs left off in the range 1 to $r$ which is $r-s$ and the number of bulbs left on to the right of $r$ which is $m-s$.

Thus, there will be at least $2 j_{l}+2 i_{m}-r$ occurrences of $t a$ and at least $l+(r-s)+(m-s)$ occurrences of $a$, giving a lower bound of $2 j_{l}+2 i_{m}+l+m-2 s=D^{\prime}(w)$ for the length of a minimal length representative of $w$.

Thus we have shown that the length of $w$ with respect to the automata generating set $\{a, t a\}$ is exactly $D^{\prime}(w)$.

As with the wreath product generating set, minimal length representatives for group elements in $L_{2}$ are not generally unique, since there are $2^{u}$ choices about whether or not the generator $a$ is applied on the first or second visit to a bulb for the $u$ bulbs that are visited twice and need an application of $a$ to leave them in their correct final state.

\section{Properties of the Cayley graphs of lamplighter groups as automata GROUPS}

3.1. Dead end words. In [1], we found that $L_{2}$ contained words which were dead end words with respect to the wreath product generating set, meaning that a geodesic ray from the identity to such a word could not be extended further. We show that $L_{2}$ also contains dead end words with respect to the automata generating set $\{a, t a\}$.

Definition 3.1. A word $w$ in a finitely generated group $G$ is a dead end word with respect to a finite generating set $X$ for $G$ if the word length $|w|=n$ and $|w x| \leq n$ for all generators $x$ in $X \cup X^{-1}$.

Such words are called dead end words because a geodesic ray in the Cayley graph $\Gamma(G, X)$ from the origin to $w$ cannot be extended beyond $w$. Note that in groups such as $L_{2}$ (with 
either generating set under consideration) where all relators are of even length, $|w x|$ will be necessarily $n-1$ for dead end words.

There are different "strengths" of dead end behavior, measured by the notion of depth.

Definition 3.2. A word $w$ in a finitely generated group $G$ is a dead end word of depth $k$ with respect to a finite generating set $X$ if $k$ is the smallest integer with the following property. If the word length of $w$ in $n$, then $\left|w x_{1} x_{2} \ldots x_{l}\right| \leq n$ for $1 \leq l \leq k$ and all choices of generators $x_{i} \in X \cup X^{-1}$.

A dead end word is a place from which is impossible to make immediate progress away from the identity in the Cayley graph $\Gamma(G, X)$. The depth of a dead end word reflects how far it may be necessary to travel before being able to move further away from the identity.

We show in [3] that all dead end words in Thompson's group $F$ with respect to the finite generating set $\left\{x_{0}, x_{1}\right\}$ have depth two, and in [1] that there are dead end words of arbitrary depth in the lamplighter groups $L_{n}$ with respect to the wreath product generating sets. The results of [1] extend to a larger class of wreath products as well. We now show that the results of [1] can be extended to the automata generating set of $L_{2}$.

Theorem 3.3. The lamplighter group $L_{2}$ contains dead end words of arbitrary depth with respect to the generating set $\{a, t a\}$.

Proof. We show that the family of words which are dead end elements with respect to the wreath product generating set are also dead end elements with respect to the automata generating set. These are the words $d_{m}$ which have all bulbs at positions within $m$ of the origin illuminated, and the cursor at the origin. Thus, $d_{m}=a_{0} a_{1} a_{2} \ldots a_{m} a_{-1} a_{-2} \ldots a_{-m}$ as pictured in Figure 2. These words have length $6 m+1$ and since the cursor is left at the origin, the exponent sum for $t a$ will be zero. Thus, both the right first and left first normal forms give rise to minimal length representatives.

As in [1, it is easily seen that these are dead end elements:

- $d_{n} a$ has one fewer illuminated bulb, and thus $D^{\prime}\left(d_{n} a\right)=D^{\prime}\left(d_{n}\right)-1$, and

- in $d_{n}(t a)^{ \pm 1}$, the cursor is at position \pm 1 with one bulb now off, and again $D^{\prime}\left(d_{n}(t a)^{ \pm 1}\right)=D^{\prime}\left(d_{n}\right)-1$.

To see that the depth is at least $m$, we note the following. A word with any possible configuration of bulbs illuminated only between $-m$ and $m$ with the cursor remaining anywhere between $m$ and $m$ will lie in the $6 m+1$ ball. Thus a path from $d_{m}$ to a point in the $6 m+2$ ball will have length at least $m$.

3.2. Seesaw words. Seesaw words are words for which a generator and its inverse reduce word length, and for which subsequent reductions in word length only occur through 


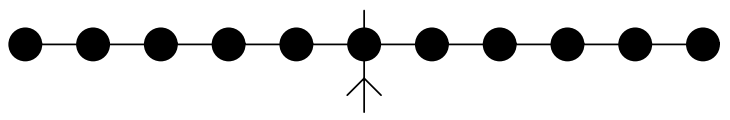

Figure 2. The dead-end word $d_{5}$, with all bulbs from -5 to 5 illuminated and the cursor at the origin.

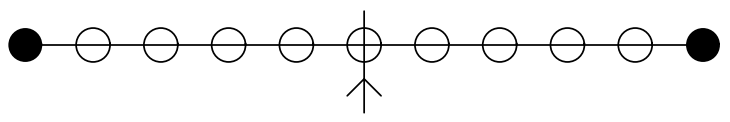

FiguRE 3. The seesaw word $w_{5}=a_{-5} a_{5}$, which has the cursor at the origin and has length 22 with respect to the automata generating set $\{a, t a\}$.

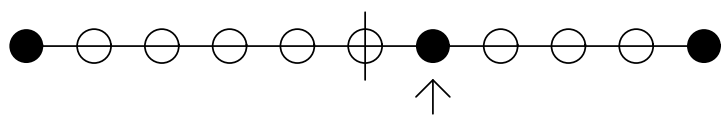

Figure 4. The word $w_{5}(t a)=a_{-5} a_{1} a_{5} t$ of length 21 with respect to the automata generating set $\{a, t a\}$.

successive applications of that generator. These words pose a difficulty for the construction of canonical minimal length representatives for elements. Thompson's group $F$ in the standard finite generating set [2], the lamplighter groups $L_{n}$ in the wreath product generating set, and a wide class of wreath products [1] contain seesaw words. Here, we also find seesaw words in $L_{2}$ with respect to the automata generating set $\{a, t a\}$.

Definition 3.4. A word $w$ in a finitely generated group $G$ with $|w|=n$ is a seesaw word of swing $k$ with respect to a generator $g_{1}$ in a generating set $X$ if the following conditions hold.

(1) The generator $g_{1} \in X$ satisfies $\left|w g_{1}^{ \pm 1}\right|=|w|-1$, and for all other $h \in X$, we have $\left|w h^{ \pm 1}\right| \geq|w|$.

(2) Additionally, $\left|w g_{1}^{l}\right|=\left|w g_{1}^{l-1}\right|-1$ for integral $l \in[1, k-1]$ and $\left|w g_{1}^{l-1} h^{ \pm 1}\right| \geq\left|w g_{1}^{l-1}\right|$ for all $h \neq g_{1} \in X$, and the analogous condition for $w g_{1}^{-1}$ is also satisfied.

Theorem 3.5. The lamplighter group $L_{2}$ contains seesaw words of arbitrary swing with respect to the generator ta from its automata generating set.

Proof. As in [1], we consider the words $w_{n}=a_{n} a_{-n}$, which have length $4 n+2$. The word $w_{5}$ is pictured in Figure 3. For a given $n$, this word consists of turning on two bulbs, a single bulb in each direction at distance $n$ from the origin and then returning the cursor to origin.

Right multiplication by $a=a^{-1}$ increases the length of $w$ by one to $4 n+3$. We now show that right multiplication by $(t a)^{ \pm 1}$ decreases the word length of $w_{n}$. 
(1) Right multiplication by ta moves the cursor to position 1 and illuminates the light bulb at that position. We obtain the expression $a_{-n} a_{n}(t a)=a_{-n} a_{1} a_{n} t$ which has word length $4 n+1$ with respect to the automata generating set.

(2) Right multiplication by $(t a)^{-1}$ illuminates the bulb at the origin, and then moves the cursor to position -1 . We obtain the expression $a_{n} a_{-n}(t a)^{-1}=a_{-n} a_{0} a_{n} t^{-1}$ which has word length $4 n+1$ with respect to the automata generating set.

The same analysis shows that until the cursor is in the position of the rightmost illuminated bulb, the generator $t a$ will reduce the word length of $w_{n}(t a)^{k}$, while the generator $a$, which illuminates an additional bulb, will increase word length. Once the cursor reaches the rightmost illuminated bulb, the generator $a$ will decrease word length. Similarly, the words $w_{n}(t a)^{-k}$ are reduced in length only by further applications of $(t a)^{-1}$ for $k=1 \ldots n-1$, so we have exhibited seesaw words of swing $k$, as desired.

\section{REFERENCES}

[1] Sean Cleary and Jennifer Taback. Dead end words in lamplighter groups and other wreath products. Submitted for publication.

[2] Sean Cleary and Jennifer Taback. Seesaw words in Thompson's group F. Submitted for publication.

[3] Sean Cleary and Jennifer Taback. Combinatorial properties of Thompson's group. Transactions of the $A M S$, to appear.

[4] Rostislav I. Grigorchuk and Andrzej Żuk. The lamplighter group as a group generated by a 2-state automaton, and its spectrum. Geom. Dedicata, 87(1-3):209-244, 2001.

\section{Sean Cleary}

Department of Mathematics

The City College of New York

City University of New York

New York, NY 10031

E-mail: cleary@sci.ccny.cuny.edu

Jennifer Taback

Department of Mathematics and Statistics

University at Albany

Albany, NY 12222

E-mail: jtaback@math.albany.edu 